\title{
Hyperprogression under immunotherapy: a new form of immunotherapy response? - a narrative literature review
}

\author{
Miaozhen Lin ${ }^{1}$, Ben G. L. Vanneste ${ }^{2}$, Qiwen Yu ${ }^{3}$, Zebin Chen ${ }^{3}$, Jiayu Peng ${ }^{3}$, Xiuyu Cai ${ }^{1}$ \\ ${ }^{1}$ Department of VIP Impatient, Sun Yat-sen University Cancer Center, Guangzhou, China; ${ }^{2}$ Department of Radiation Oncology (MAASTRO \\ Clinic), GROW-School for Oncology and Developmental Biology, Maastricht University Medical Center, Maastricht, The Netherlands; \\ ${ }^{3}$ Department of Oncology, The First Affiliated Hospital of Guangdong Pharmaceutical University, Guangzhou, China \\ Contributions: (I) Conception and design: X Cai; (II) Administrative support: BGL Vanneste, X Cai; (III) Provision of study materials or patients: X \\ Cai; (IV) Collection and assembly of data: Z Chen, Q Yu; (V) Data analysis and interpretation: Mi Lin, J Peng; (VI) Manuscript writing: All authors; \\ (VII) Final approval of manuscript: All authors. \\ Correspondence to: Xiuyu Cai. Department of VIP Impatient, Sun Yat-sen University Cancer Center, 651 Dongfeng Road East, Guangzhou 510060 , \\ China. Email: caixy@sysucc.org.cn.
}

Objective: Update the last known review, and summarize the definitions, diagnostic criteria, reported risk factors, possible mechanisms and potential biomarkers of hyperprogressive disease (HPD) under immunotherapy.

Background: Immunotherapy is a relatively new systemic therapy adding a new method of treatment of especially advanced cancer patients. In a variety of immunotherapies, however, an unexpected acceleration of tumor growth, known as HPD, is observed in approximately $30 \%$ of patients after immune checkpoint inhibitor (ICI) treatment. HPD has a deleterious survival effect on patients and represents an urgent issue for both clinicians and patients. Existing literature has reviewed and summarized the definition, diagnostic criteria, reported risk factors and possible mechanisms of hyperprogression. However, with the gradual deepening of the exploration of HPD, researchers have made significant breakthroughs in elucidating the mechanism and mechanism of HPD and exploring biomarkers.

Methods: The search was conducted on Google Scholar and PubMed in January and May of 2021. We searched among English papers with no limitation on the publication year. We have included retrospective studies, case reports and basic researches related to HPD in the collection, we also referred to some review articles on HPD in recent years. A qualitative-interpretive approach was used for data extraction.

Conclusions: HPD is considered to be an acceleration of tumor growth after ICI treatment that is not only due to immune infiltration but also due to real disease progression, with an incidence of about 4-30\% in all retrospective published studies to date. Currently, the most widely used criteria of HPD contain Response Evaluation Criteria in Solid Tumors (RECIST) and tumor growth rate (TGR) or tumor growth kinetics. The common risk factors and underlying mechanisms of HPD have not yet been fully elucidated. However, based on the poor prognosis of HPD, there have been many advances in the exploration of biomarkers in recent years, like the prediction of HPD, such as LDH levels of peripheral blood, liquid biopsy, and radiomics, etc.

Keywords: Immunotherapy; hyperprogressive disease (HPD); hyperprogression (HP)

Submitted Apr 22, 2021. Accepted for publication Jul 26, 2021.

doi: $10.21037 /$ tlcr-21-575

View this article at: https://dx.doi.org/10.21037/tlcr-21-575 


\section{Introduction}

The treatment of especially metastatic cancer patients has been dramatically improved by the introduction of immunotherapy. There are already 3 immune checkpoint inhibitors (ICIs) that target cytotoxic $\mathrm{T}$ lymphocyteassociated molecule-4 (CTLA-4), programmed cell death receptor-1 (PD-1), and programmed cell death ligand-1 (PD-L1) are the most widely studied and recognized. It has been proven that ICIs can increase the overall survival (OS) in variety of malignancies, such as melanoma, nonsmall cell lung cancer (NSCLC), renal cancer, head and neck squamous cell carcinoma (HNSCC), urothelial bladder cancer, and endometrial stromal sarcoma (1-3). However, a small subset of patients on immunotherapy may not benefit at all from immunotherapy, instead, they experience a faster and more aggressive progression of the tumor than expected, with a dramatic acceleration of the disease, which is referred to as hyperprogression (HP) or hyperprogressive disease (HPD). Patients with HPD could suffer a deleterious survival effect and significantly shorter OS, suggesting that HP should be managed as fulminant toxicity and needs to be considered before immunotherapy is initiated. Existing literature has reviewed and summarized the definition, diagnostic criteria, reported risk factors and possible mechanisms of HP. However, with the gradual deepening of the exploration of HPD, researchers have made significant breakthroughs in elucidating the mechanism and mechanism of HPD and exploring biomarkers. On this basis, we reviewed and summarized the definition, incidence, diagnostic criteria, reported risk factor and possible mechanisms of HPD in recent years, and briefly introduced a novel perspective that few people mentioned: the potential biomarkers of HPD. We present the following article in accordance with the Narrative Review reporting checklist (available at https://dx.doi. org/10.21037/tlcr-21-575).

\section{Methods and results}

The search was conducted on google scholar and PubMed in January and May of 2021. We searched among English papers with no limitation on the publication year. We have included retrospective studies, case reports, basic research articles related to HPD in the collection, we also refer to some review articles, commentary articles on HPD in recent years. A qualitative-interpretive approach was used for data extraction.
We have collected 61 references in total, 24 of which are clinical articles, including retrospective studies, cohort studies, case reports etc., 15 of which are basic research articles, 9 of which are review articles or commentary articles, and the rest of which are some guidelines and consensus in the oncology field, and reference articles that needed to explain definitions.

\section{Discussion}

\section{Appearance, definitions, and diagnosis of HPD}

HPD is considered to be an acceleration of tumor growth after ICI treatment that is not only due to immune infiltration but also due to real disease progression $(4,5)$. It was first reported in a retrospective study published by Lahmar et al. in 2016. They performed a retrospective study of 89 NSCLC patients treated by ICIs, calculated the tumor growth rate [TGR: the log-scale calibrated change in the sum of the volumes of the target lesions according to Response Evaluation Criteria in Solid Tumors (RECIST) 1.1 criteria per month] before and after ICIs treatment, and found that $\triangle$ TGR (difference between TGR during ICIs and TGR at baseline) was $<0$ in 79 patients and $>0$ in 20 patients; and among these 20 patients, 9 had a $\Delta$ TGR $>50 \%$, meaning that tumor volume increased at least $50 \%$ during immunotherapy (6). In the same year, Chubachi et al. also reported a case of rapid lung cancer progression after 3 cycles of nivolumab treatment and described it as a "disease flare" (7). Later, in 2017, Champiat and his colleagues defined HPD as patients who have been assessed with disease progression by the RECIST for the first evaluation with at least a 2 -fold increase in TGR before and after receiving immunotherapy. Subsequently, an increasing number of studies have confirmed the existence of the HPD phenomenon in many types of tumors, like NSCLC, esophageal squamous carcinoma, melanoma, soft tissue sarcoma, etc. (8-11). HP has become an increasingly recognized phenomenon.

Despite its growing incidence, the diagnostic criteria for HPD have not yet been standardized. Clinicians have previously evaluated the activity and efficacy of new cancer therapeutics in solid tumors by immune-related response criteria (irRC), RECIST, immune RECIST (iRECIST), immune-related RECIST (irRECIST), or immune-modified RECIST (imRECIST) (12-14). We have compiled these criteria in Table 1, all of these criteria divide efficacy into 4 levels (Figure 1): complete response (CR), 
Table 1 Overview of radiological criteria in immuno-oncology

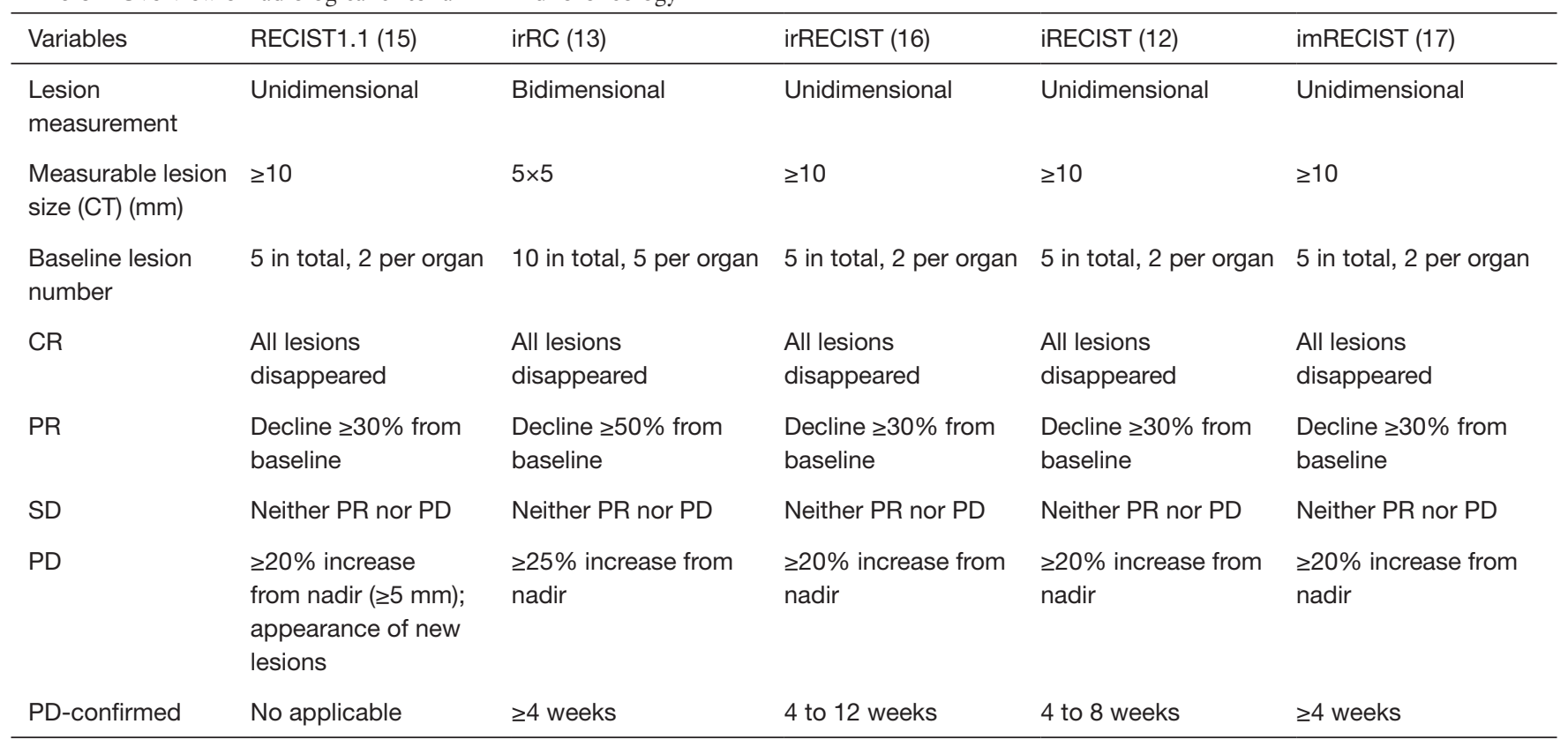

RECIST, Response Evaluation Criteria in Solid Tumors; irRC, immune-related response criteria; iRECIST, immune RECIST; irRECIST, immune-related RECIST; imRECIST, immune-modified RECIST; CT, computed tomography; CR, complete response; PR, partial response; $\mathrm{SD}$, stable disease; PD, progressive disease.

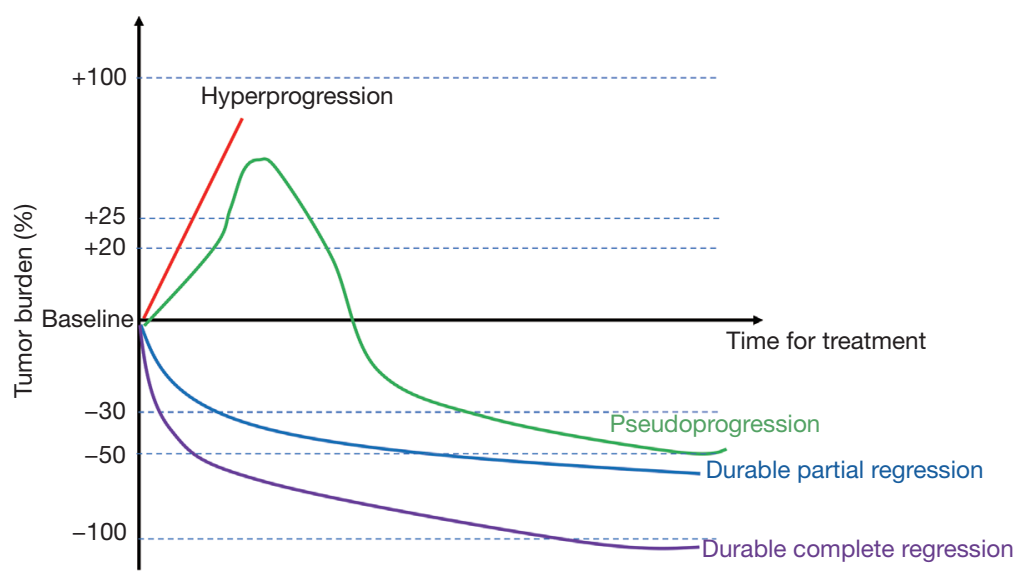

Figure 1 Patterns of response to immunotherapy.

partial response (PR), stable disease (SD), and progressive disease (PD). In PD, several subclassifications are not mentioned in detail in RECIST: confirmed PD; HP, and pseudoprogression.

Currently, the most widely used criteria contain RECIST and TGR or tumor growth kinetics [TGK: the change in the sum of the longest diameters (SLD) of the target lesions according to RECIST 1.1 criteria per month]
(Figure 2). For example, in the study of Kanjanapan et al., HPD was considered to be RECIST-defined PD at first assessment and a more than 2-time TGR increase after the initiation of immunotherapy (18). Meanwhile, SaâdaBouzid $e t a l$. defined HP as TGK ratio $\left(\mathrm{TGK}_{\mathrm{R}}\right.$ : TGK post-immunotherapy/TGK pre-immunotherapy) $\geq 2$, and these authors reported a $29 \%$ incidence of HPD (19). The common requirement of TGR and TGK measurement is 


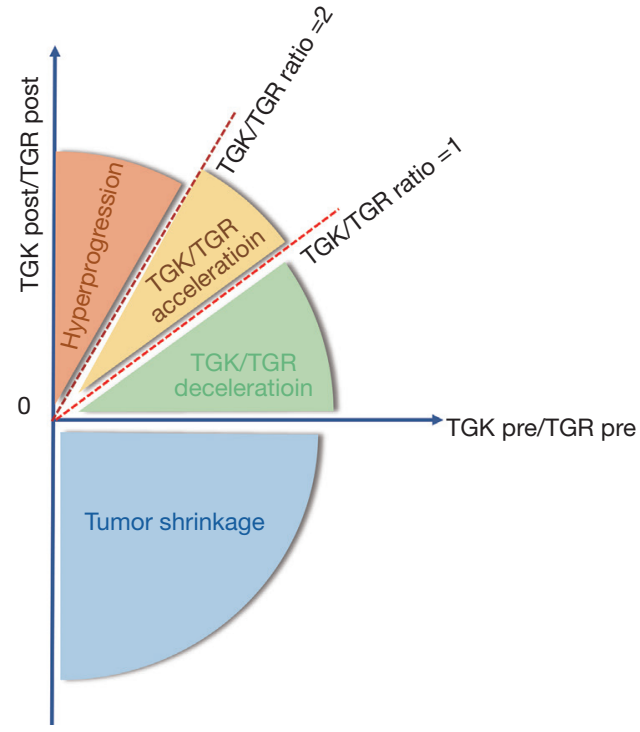

Figure 2 TGK/TGR criteria in defining HPD. TGK, tumor growth kinetics; TGR tumor growth rate; HPD, hyperprogressive disease.

a comparison of the variation of target lesions before and after immunotherapy is initiated, which mainly depends on computed tomography (CT) scan. As Ferrara et al., they defined HPD as disease progression at the first evaluation with $\triangle$ TGR exceeding $50 \%$ (20). However, the exclusive use of a radiological definition of HPD could easily misdiagnose pseudoprogression as HP (21), which could lead to an overestimate. Moreover, in some cases of rapid clinical decline, performing a confirmatory CT scan may not be feasible. Therefore, some authors propose adding other clinical criteria to the diagnostic criteria for HPD. For instance, Kato et al. suggest 3 criteria to define HPD: time to treatment failure (TTF) $<2$ months; and a tumor burden increase of $50 \%$ with a progression rate increase of at least 2 times (22). In addition to these, Lo Russo et al. also proposed the following diagnostic criteria: $\geq 2$ new lesions appearing in 1 already-involved organ or new involved organs appearing at the first radiological evaluation after the initiation of immunotherapy, and an Eastern Cooperative Oncology Group (ECOG) score decrease of 2 points or more within the first 2 months of treatment (23). However, too great a consideration for clinical factors can confer disadvantages, as progressive PD may be mistaken for HPD; this contradiction may be part of the reason why there is still no consensus on the diagnostic criteria for HPD.
Incidence, risk factors, prognosis, and potential biomarkers

\section{Incidence}

HPD is not only caused by immunotherapy. One post hoc analysis from the OAK trial suggested that rapid progression is a common phenomenon that co-occurs with chemotherapy and ICIs. Likewise, Gandara et al. found in their study that the atezolizumab and docetaxel groups had a similar proportion of patients with rapid progression, meaning that the rapid progression after baseline was not specific to PD-L1 inhibitor therapy (24). For radiotherapy, the correlation between HPD and radiotherapy is more ambiguous, Saâda-Bouzid et al. noticed that almost all cases of HPD during immunotherapy in their study occurred in patients who had at least one locoregional recurrence in an irradiated field, suggesting that radiotherapy might play a role in the process of HPD (19). And a recent case report also reported a 42-year-old woman with stage IV renal clear cell carcinoma, experienced HPD during immunotherapy after receiving stereotactic body radiation therapy (SBRT) (25). However, there are no reports of HPD cases during pure radiotherapy.

Overall, HPD is not a unique phenomenon of immunotherapy, while in immunotherapy, the incidence of HPD is higher, especially when PD-1 or PD-L1 inhibitor is used. According to published relevant research data, the prevalence of immunotherapy-related HPD ranges from a few percent to about $30 \%(5,26)$. Champiat et al. reported an incidence of HPD of $9 \%(12 / 131)$, and this ratio may be underestimated, as some patients could not be evaluated due to clinical progression; thus, the true incidence of HPD might be higher than that reported (4). Additionally, the incidence of HPD may also be related to the type of tumor. Saâda-Bouzid et al. reported a $29 \%$ incidence of HPD in hypopharyngeal squamous cell carcinoma (19); the incidence of HPD in other cancers has been reported, with gastric cancer having an HPD incidence of $11 \%$ (27), NSCLC a range from $13.8 \%$ to $25.7 \%(20,23,28)$, melanoma 6-34\%, gynecological cancer $16 \%$, cutaneous squamous cell carcinoma $9 \%$, renal cancer $5-7 \%$, colorectal cancer 6\%, and urothelial cancer 6\% (29).

\section{Risk factor}

HPD has been putatively associated with several risk factors, including age ( $>65$ syears), genomic alterations, and metastasis burden (the number of sites of metastatic disease), or locoregional recurrence $(4,19,23,24,29,30)$. 


\section{Age ( $>65$ years)}

Many studies have shown that older patients have a higher chance of developing HPD. Champiat et al. observed that patients with HPD were older than those without HPD $(\mathrm{P}=0.07)$, and suspected that old age might be related to a different immunological background or higher concentrations of inflammatory cytokines (4). Meanwhile, Refae et al. also reported that HPD is significantly associated with age $\geq 70$ years $(25 \%$ versus $6 \%$; $\mathrm{P}=0.025)$ (31).This may be related to immune cells, chemokines, phagocytosis, and the weakened ability of intracellular antigens in elderly patients, but the specific mechanism is unknown.

\section{Genomic alterations}

Murine double minute 2/4 proto-oncogene (MDM2/4) family amplification and epidermal growth factor receptor (EGFR) aberrations are the most widely studied alterations in HPD. One study that analyzed 11 patients with multiple tumors who experienced HP in a multi tumor cohort demonstrated that rs2282055 (PD-L1) and rs1870377 [vascular endothelial growth factor receptor 2 (VEGFR2)] variations have a significant and independent influence on the occurrence of HPD (31). Another preliminary report by Tawbi and colleagues suggested that liposarcoma patients, who commonly harbor MDM2 amplification, may benefit from immunotherapy (PR rate of 11\%) (32). Meanwhile, Kato et al. found that 6 patients with MDM2/ MDM4 expansion had TTF in less than 2 months; with 4 of them meeting the definition of HP; the tumor volume increased by $55 \%$ to $258 \%$, and the rate of progression increased by $2.3,7.1,7.2$, and 42.3 times from the baseline, respectively. Patients with MDM2/MDM4 amplification showed significantly accelerated tumor growth compared with that observed before treatment (30). In the study by $\mathrm{Ye}$ et al. (33), even though no statistically significant association was observed between MDM2, MDM4, or EGFR amplification and the efficacy of checkpoint inhibitors, it was nonetheless observed that patients with MDM2/4 or EGFR amplification were more prone to developing HP and had a lower chance of disease control.

\section{Metastasis burden and locoregional recurrence}

A retrospective study published by Ferrara et al. found that NSCLC patients with multiple metastases at baseline tended to experience HP. They found in their study that patients with HP were significantly more likely to have had 2 or more metastases before receiving PD-1/PDL1 therapy (among patients with $\geq 2$ metastases, $62.5 \%$ developed HP, P=0.006) (20). Saâda-Bouzid et al. also found in their study that the incidence of HP was $42 \%$ in patients with metastatic cervical lymph nodes and only $26 \%$ in patients without cervical lymph nodes metastasis and that regional recurrences occurred in $90 \%$ of patients with HP, but only in $37 \%$ of patients without progression, indicating that $\mathrm{HP}$ was significantly associated with region recurrence $(\mathrm{P}=0.008)$, but not with region recurrence (19).

\section{Potential biomarkers Peripheral blood biomarkers}

For years, scientists have been looking for a non-invasive cancer biomarker, and the marker in the peripheral blood is undoubtedly one of the most convenient. Peripheral white blood cell (WBC) counts such as neutrophil count (NC), lymphocyte count (LC), the neutrophil to lymphocyte ratio (NLR), and the derived neutrophil to lymphocyte ratio [dNLR: absolute NC/(WBC - absolute NC)] have been demonstrated that can provide some clue to HPD. Kiriu et al. obeserved 19 patients treated with nivolumab, and found that NLR is rising from baseline in 5 out of 7 patients with PD. and patients with an $>30 \%$ increase in NLR were associated with a significantly shorter TTF compared with those with stable or decrease in NLR both after first cycle $(\mathrm{P}=0.014)$ and second cycle $(\mathrm{P}=0.001)$ (34). Another popular biomarker is serum lactate dehydrogenase (LDH), Sasaki et al. indicated that a high level of $\mathrm{LDH}$ was associated with HPD $(18,35)$. In a recent study, the investigator demonstrated that the risk of HPD in LDH > upper limit of normal (ULN) was 2.32 times higher than that in $\mathrm{LDH}$ $\leq \mathrm{ULN}(\mathrm{P}=0.0001)$ (36). However, some researchers hold different opinions, like Champiat et al. and Ferrara et al., they didn't find the association in find any significant differences in dNLR, WBC, NC, LC, or LDH $(4,20)$.

\section{Liquid biopsy}

As a branch of in vitro diagnosis, liquid biopsy is a noninvasive blood test to monitor circulating tumor cells (CTCs), circulating tumor DNA (ctDNA) and cellfree DNA (cfDNA) fragments released into the blood by tumors or metastatic lesions. ctDNA and cfDNA can reflect blood-based tumor mutational burden (bTMB) and chromosomal instability of cancer cells, which has been linked to poor prognosis and resistance to treatment in several malignancies. By analyzing the mutations detected in ctDNA, bTMB can be calculated to evaluate tumor response to immunotherapy, and increased TMB has been associated with a higher likelihood of immunotherapy response (37-39). More importantly, the dynamics of ctDNA may be more sensitive than radiological tests. In a study involving 125 melanoma patients, the researchers found 
that none of the patients ( $n=9)$ who were finally diagnosed with pseudoprogression did not observe a significant increase in ctDNA (even it is detectable at baseline, subsequent $c t D N A$ levels will be reduced by $>10$ times due to treatment), despite the significant increase in tumor size on imaging (40). And Weiss et al. used chromosomal number instability (CNI) score to evaluate 56 patients treated with immunotherapy across multiple tumor types, six of whom had HP, and in 5 of these 6 cases, the CNI score also predicted progression at an earlier time ( $6-9$ weeks) compared with routine imaging (41). This shows that cfDNA is expected to become a potential biomarker for predicting HPD.

\section{Radiomics}

Radiomics is an emerging field that translates medical images into quantitative data and enables these data to be extracted and applied in clinical decision-making, thereby improving diagnostic, prognostic, and predictive accuracy. In cancer research, radiomics has also shown promise in tumor diagnosis, clinical staging, response evaluation, recurrence, distant metastasis, and prognosis prediction $(42,43)$. The image information extracted by Radiomic includes but is not limited to size/shape, heterogeneity/texture, relationship with surrounding tissues, which also provides a basis for evaluating the efficacy of immunotherapy, because tumor size changes caused by tumor cell necrosis may not necessarily occur in the course of immunotherapy. Besides, Sun et al. observed a significant correlation between the genomic and radiomic characteristics $(\mathrm{P}<0.0001)$ and the abundance of $\mathrm{CD} 8$ cells as well as tumor-associated neutrophils $(\mathrm{P}=0.0079)$, which are associated with poor response to immunotherapy (44). The study published by Ji et al. also validated that radiomics could separate patients who did and did not benefit from immunotherapy, they constructed 4 radiomics models to explore that whether radiomics can use in predicting the effectiveness of immunotherapy. And the sensitivity, specificity, and area under the curve (AUC) of the best model can reach $83.3 \%, 88.9 \%$, and 0.806 respectively (42). From this point of view, radiomics may be used as one of the promising markers, through which, it may be possible to stratify people undergoing immunotherapy and screen out potential populations that may develop HPD. While more research is still needed to support this.

\section{Prognosis of HPD}

Patients with HPD may have a significantly shorter OS than other patients (Table 2). A study by Kurman et al. found that the median OS of non-HPD patients was 7.6 months, while that of HPD patients was only 4.6 months (21). Another meta-analysis reported that HPD patients were associated with the worse OS when compared to non-HPD individuals (3). However, no such difference in survival was found in patients with HPD after receiving chemotherapy, thus implicating immunotherapy as a possible factor in HPD emergence.

\section{Potential mechanisms}

Multiple mechanisms have been proposed to participate in the development of HP. We here summarize the most commonly discussed mechanisms in the recent literature (Figure 3).

\section{Massive expansion of $P D-1$ and $T$ regulatory cells}

Regulatory T (T-reg) cells are a kind of immunosuppressive cell that exist in the immunosuppressive tumor microenvironment (TME) (49). ICIs such as CTLA4 and PD-1, along with stimulating receptors like tumor necrosis factor receptor superfamily member 4 (OX40) and glucocorticoid-induced TNFR-related protein (GITR), are also expressed by T-reg cells. PD-1 works by blocking TCR and CD28 signals to inhibit the excessive activation of effector T cells (49). PD-L1 can increase the possibility of mutual PD-1 and PD-L1 signaling, allowing delicate control of immune cell homeostasis $(50,51)$. Meanwhile, the survival and function of T-reg cells in TME are dependent on T cell receptor (TCR) and CD28 signals (49), and the blockade of PD-1/PD-L1 may activate the immune-suppressive function of T-reg cells, promoting the proliferation of T-reg cells, resulting in further suppression of immunity and rapid tumor progression (49). In line with this hypothesis, Kamada et al. found that non-HPD patients exhibited a drastically reduced ratio of effector regulatory $\mathrm{T}$ (eT-reg) cells to $\mathrm{CD}^{+}{ }^{+} \mathrm{T}$ cells and the ratio of $\mathrm{Ki} 67^{+}$eT-reg cells to $\mathrm{Ki} 67^{+} \mathrm{CD} 8^{+} \mathrm{T}$ cells (mainly with $\mathrm{Ki} 67^{+}$eT-reg cells decreases) in tumor Infiltrating lymphocytes; meanwhile, HPD patients showed no significant change or just a slight increase. This clinical result was validated through in vitro and in vivo mouse models, providing strong support for the role of T reg cells in HP (27).

\section{TME}

Research shows that in HPD tumors, an immunosuppressive environment forms after ICI treatment. The presence of inflammatory cells in TME can lead to tumor escape by triggering local inflammation, remodeling matrix tissue, modifying metabolism, or promoting angiogenesis $(46,52)$. Moreover, Xiong et al. also identified that the activities of 


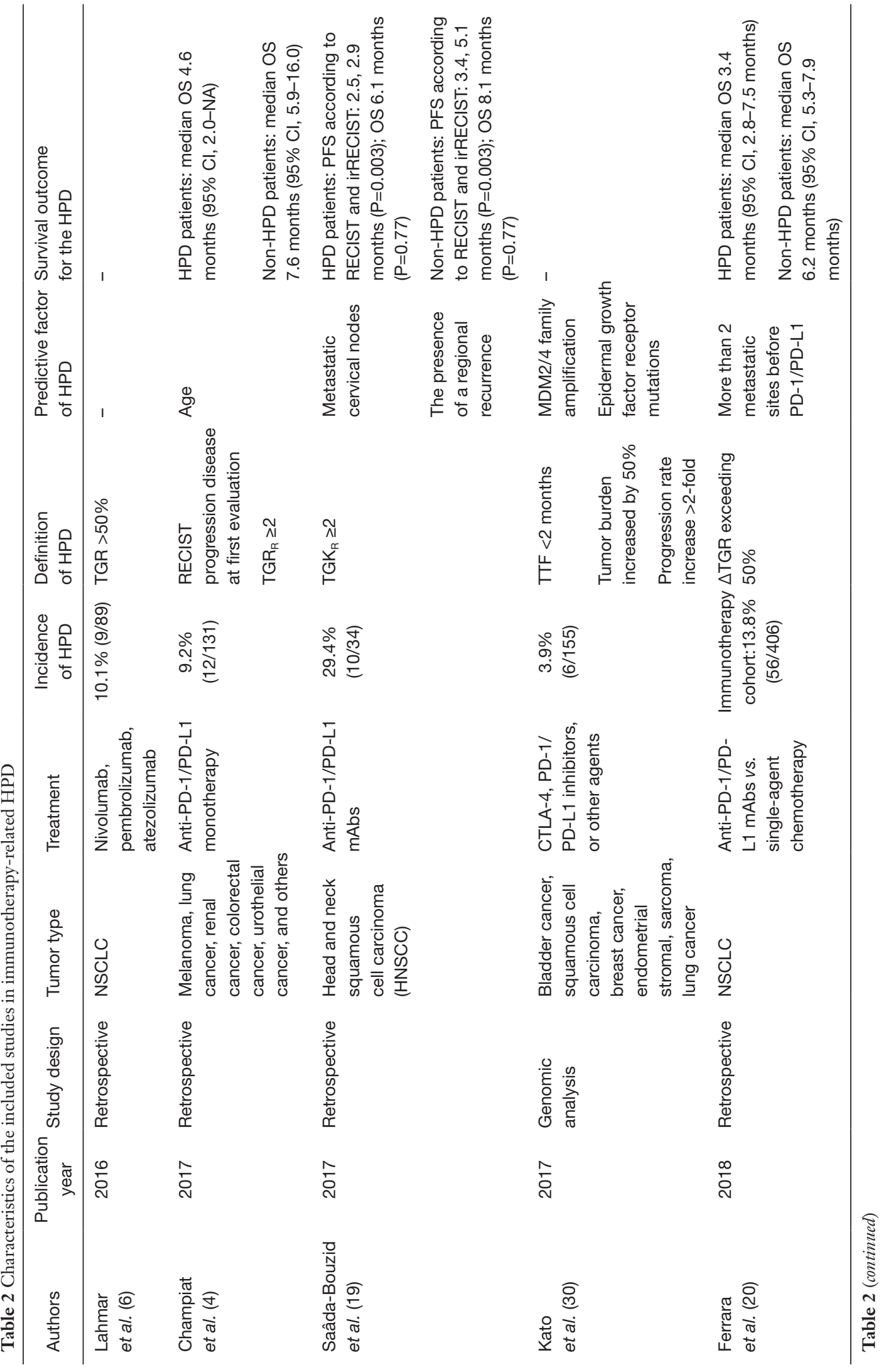




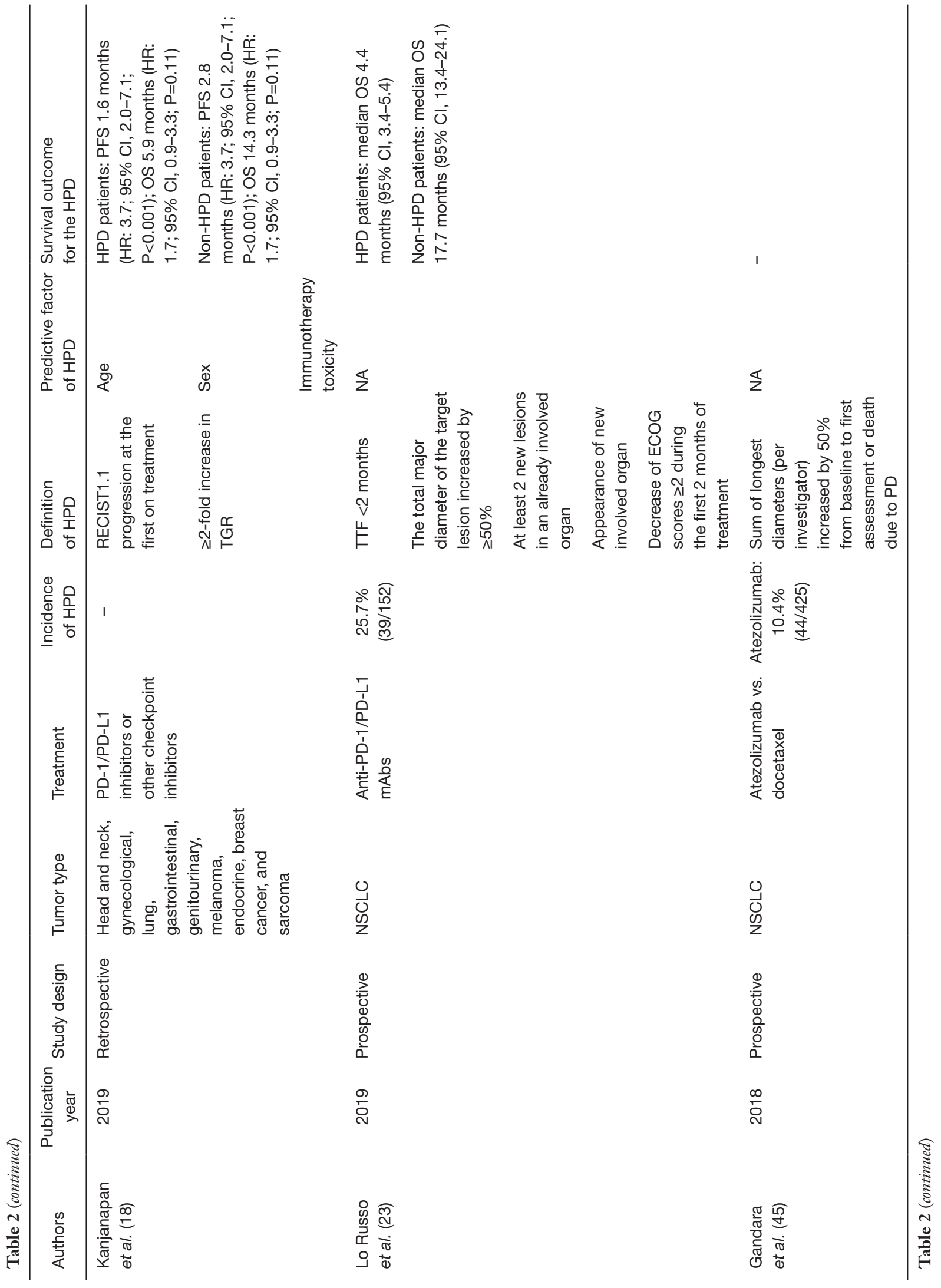




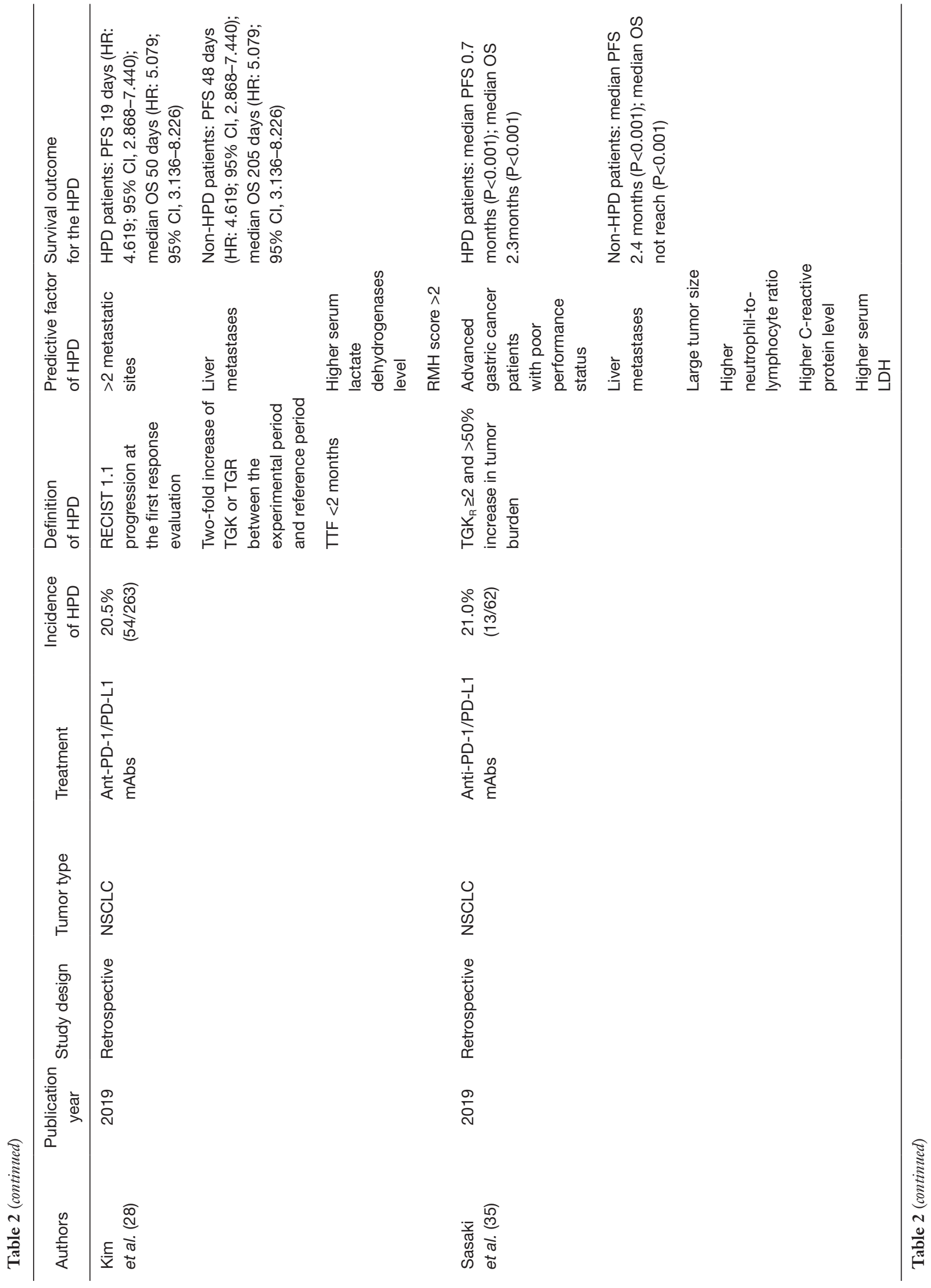




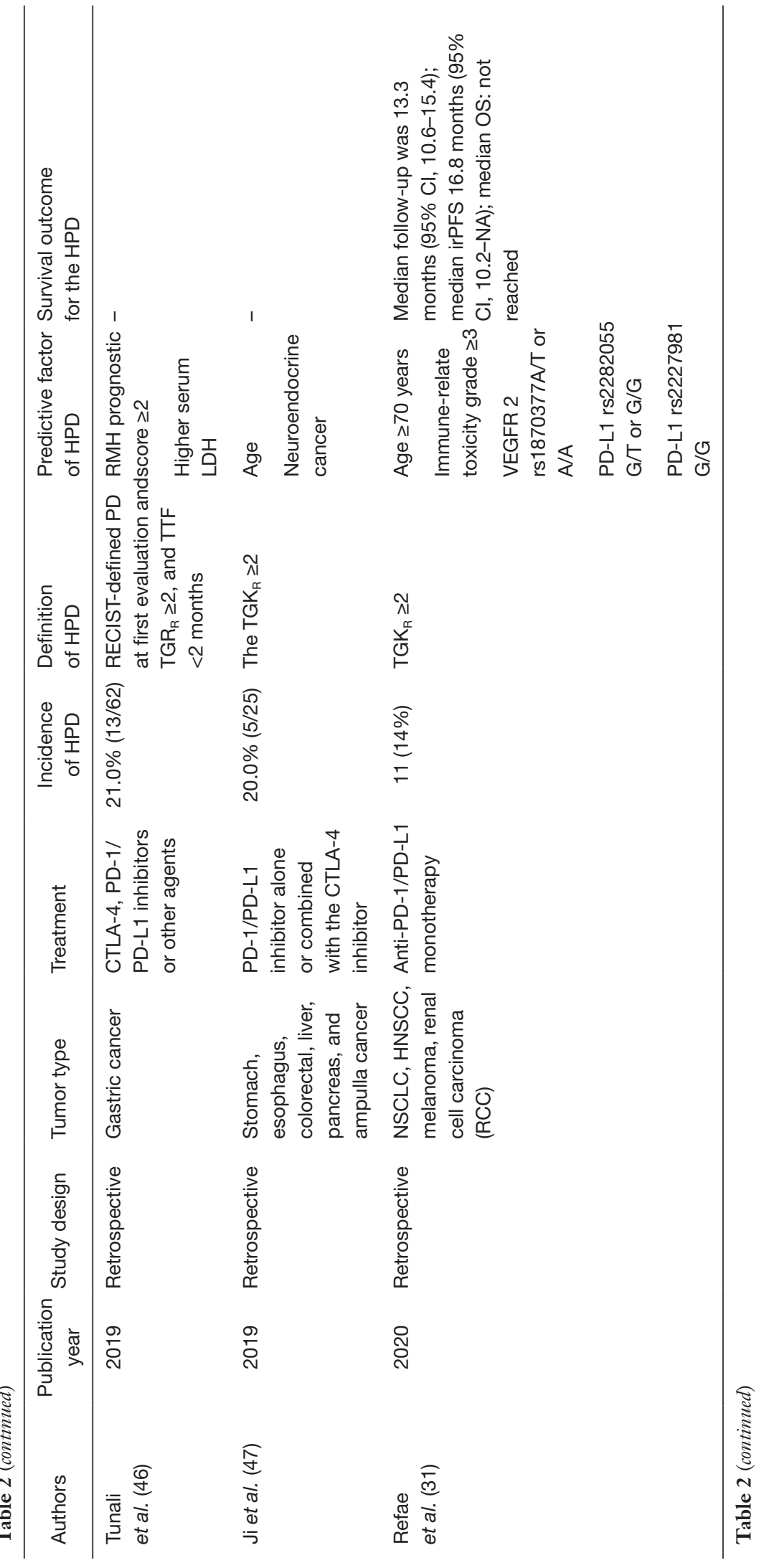




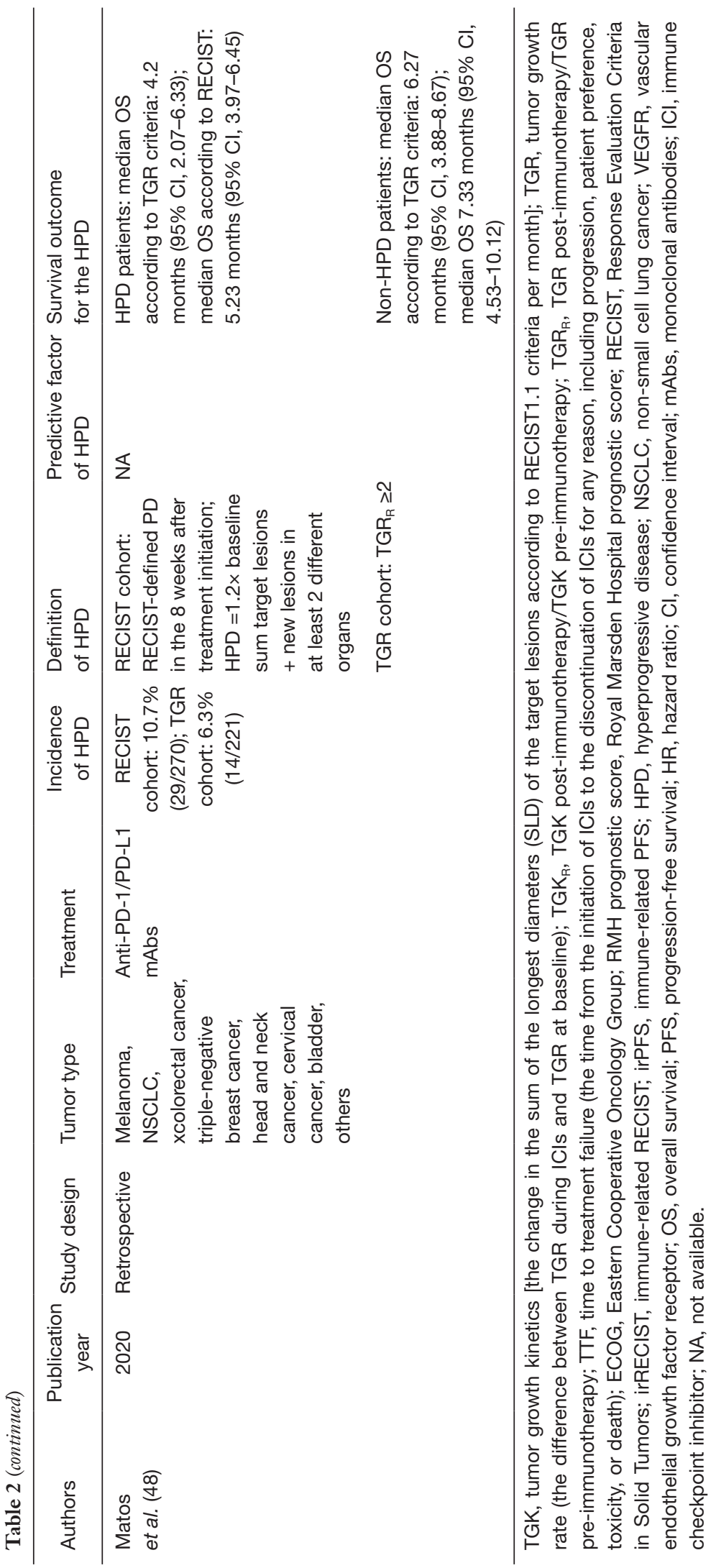




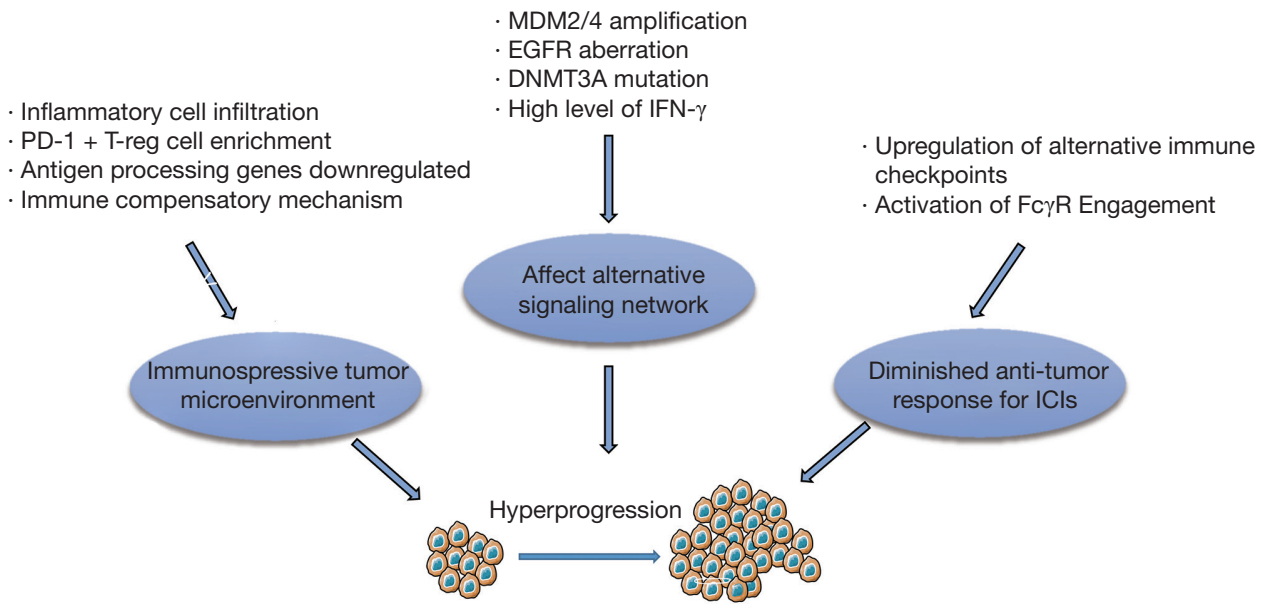

Figure 3 Potential mechanisms of HPD. T-reg cell, regulatory T cell; MDM2/4, murine double minute 2/4 proto-oncogene; EGFR, epidermal growth factor receptor; DNMT3A, DNA methyltransferase 3 alpha; IFN- $\gamma$, interferon- $\gamma$; Fc $\gamma$ R, Fc $\gamma$ receptor; HPD, hyperprogressive disease; ICIs, immune checkpoint inhibitors.

immune cell populations were significantly decreased while other immune cell populations that attenuate immune responses in the TME were upregulated in the HPD tumors after anti-PD-1 treatment (52). Another study by Lo Russo et al. retrospectively analyzed 152 patients with NSCLC who received immunotherapy and found an enrichment of tumor-associated macrophages in patients with HP. By using immunofluorescence staining, immunophenotypic macrophages were observed in patients with HP; CD163, CD33, and PD-L1 were all positive and co-expressed, and statistically showed different expression compared with non-HPD patients $(\mathrm{P}<0.0001)(23)$. In addition, vascular endothelial growth factor (VEGF) and high LDH concentration in serum may also play a part in affecting the TME. VEGF can influence the immune regulatory environment of tumors by inhibiting the maturation of myeloid and dendritic precursors and the recruiting immunosuppressive $M 2$ macrophages which may be involved in HPD (30). Finally, elevated levels of $\mathrm{LDH}$ promote hypoxia in the tumor and acidification of the extracellular environment, which may reduce ICI efficacy, leading to tumor cell proliferation, angiogenesis, and antiapoptosis (53).

\section{Specific genomic alterations}

Particular mutation could cause HPD (30). Xiong et al. analyzed post-therapy HPD tumors with mutation analysis and identified 11 genes with deleterious mutations, including TRPC4, POTEE, FBN2, KMT2C, FUT10, $P Q B P 1, T S C 2$, etc. (52). Kato et al. found that patients with
$M D M 2 / M D M 4$ amplification or DNMT3A gene mutation were more likely to develop HP (30). Ye et al. found that patients with MDM2/4 or EGFR amplification are more likely to developed HP. MDM2 can directly interact with p53, induce its degradation and limit its function (33), JAK-STAT signaling activated by ICIs can also increase interferon regulatory factor (IRF)-8, which can bind to MDM2 promoter and induce MDM2 expression (54). Moreover, MDM2 expression can regulate VEGF expression in some cancers, and this may contribute to a variety of MDM2 functions in promoting cancer growth and metastasis (55). EGFR activation has also been associated with the upregulation of the checkpoints CTLA-1, PD-1, and PD-L1, which can drive immune escape (56). Epidermal growth factor (EGF) signal transduction has also been shown to induce posttranslational modification of PD-L1, which might be associated with poor prognosis and metastasis (29).

\section{Activation of Fcy receptor ( $F c \gamma R$ ) engagement}

ICIs could likely promote tumor growth through stimulating PD-1 on myeloid cells. Du et al. found that tumors treated with PD-1 inhibitor displayed elevated Ki-67 expression, reduced cleaved caspase- 3 expression, increased proliferation, and decreased apoptosis, suggesting that PD-1 blockade may enhance cancer viability and contribute to the procession of HPD (57). However, this hypothesis is still in its infancy, and more cases are needed to support it. In another study by Knorr and colleagues (58), infiltrating leukocytes were evaluated, and the analysis showed that 
inhibitors of Fc $\gamma$ R IIB (Fc $\gamma$ RIIB) in tumor draining lymph node (TDLN) and the TME, Fc $\gamma \mathrm{R}$ expression within tumors, and Fc $\gamma$ RIIA and Fc RIIIA in the TDLN were significantly increased, indicating that the mechanism of HP may be Fc-receptor dependent. Indeed, in 2015, Dahan et al. had already demonstrated that activation of $\mathrm{Fc} \gamma \mathrm{R}$ involvement could reduce the antitumor response of antiPD-1 antibodies (59). In 2018, Zhang et al. designed 2 antiPD-1 monoclonal antibody with the same specificity, one which bound to FcR and the other which did not, and the result showed that the latter does prevent tumor growth, while the former can cause macrophage PD-1-T cell crosslink and phagocytosis of PD-1 and T-cells (60).

In this mechanism, FcyRIIB might be the key receptor, as 2141-V11 and Fc $\gamma$ RIIB have the strongest binding, while FcyRIIB cross-linking can strengthen myeloid cell signal PD-1 $(58,61)$. In addition, the repolarization of tumorassociated macrophages is dependent on FcyRIIB, and FcR on specific M2-like intratumoral macrophages may result in these cells being reprogrammed to promote tumorigenesis, the discovery of which may provide insight into the mechanism of HP $(1,61)$.

\section{Conclusions}

HP is a distinct response pattern in immunotherapy, with an incidence of about 4-30\% in all retrospective published studies to date, but with no consensus concepts, predictors, diagnosis criteria, or clear mechanisms related to this condition. Now we looking back at these published studies, there are still limitations in the exploration of HPD, such as the limitations in cohort size, lack of control, and different evaluation methods so on. Besides, there are also many confusions about HPD: why does HPD perform prominently in immunotherapy? Is immunotherapy really completely contraindicated for patients with HPD? Is it possible to reverse HPD?

For now, $\mathrm{HP}$ is as much of a challenge as it is an opportunity in immunotherapy, as and the molecular and clinical understanding of HPD is still in its preliminary stages, and it has been reported that HP under immunotherapy may be deleterious to survival, for patients who already experience HP occurs, the current immunotherapy regimen should be stopped first, relieve life threats for patients and choose a treatment-sensitive treatment plan for the patient at the appropriate time. But for patients who have already experienced HPD, the follow-up treatment is not conclusive. Therefore, before exploring what to do after it has happened, stratify the expected benefits of patients, thereby improving the accuracy of predicting individual response to treatment, and identify especially the patients who are at risk for HPD, and select them for an alternative approach is more important in current status. Exciting thing is there have been many promising markers for the prediction of HPD, such as LDH levels of peripheral blood, liquid biopsy, and radiomics, I believe in the future there will be more studies appear jointly to open the veil of HPD under immunotherapy.

\section{Acknowledgments}

The authors appreciate the academic support from AME Cancer Immunotherapy Collaborative Group.

Funding: None.

\section{Footnote}

Reporting Checklist: The authors have completed the Narrative Review reporting checklist. Available at https:// dx.doi.org/10.21037/tlcr-21-575

Conflicts of Interest: All authors have completed the ICMJE uniform disclosure form (available at https://dx.doi. org/10.21037/tlcr-21-575). The authors have no conflicts of interest to declare.

Ethical Statement: The authors are accountable for all aspects of the work in ensuring that questions related to the accuracy or integrity of any part of the work are appropriately investigated and resolved.

Open Access Statement: This is an Open Access article distributed in accordance with the Creative Commons Attribution-NonCommercial-NoDerivs 4.0 International License (CC BY-NC-ND 4.0), which permits the noncommercial replication and distribution of the article with the strict proviso that no changes or edits are made and the original work is properly cited (including links to both the formal publication through the relevant DOI and the license). See: https://creativecommons.org/licenses/by-nc-nd/4.0/.

\section{References}

1. Frelaut M, Le Tourneau C, Borcoman E. Hyperprogression under Immunotherapy. Int J Mol Sci 2019;20:2674. 
2. Denis M, Duruisseaux M, Brevet M, et al. How Can Immune Checkpoint Inhibitors Cause Hyperprogression in Solid Tumors? Front Immunol 2020;11:492.

3. Kim JY, Lee KH, Kang J, et al. Hyperprogressive Disease during Anti-PD-1 (PDCD1) / PD-L1 (CD274) Therapy: A Systematic Review and Meta-Analysis. Cancers (Basel) 2019;11:1699.

4. Champiat S, Dercle L, Ammari S, et al. Hyperprogressive Disease Is a New Pattern of Progression in Cancer Patients Treated by Anti-PD-1/PD-L1. Clin Cancer Res 2017;23:1920-8.

5. Sabio E, Chan TA. The good, the bad, and the ugly: hyperprogression in cancer patients following immune checkpoint therapy. Genome Med 2019;11:43.

6. Lahmar J, Mezquita L, Koscielny S, et al. Immune checkpoint inhibitors (IC) induce paradoxical progression in a subset of non-small cell lung cancer (NSCLC). Ann Oncol 2016;27:vi416-vi454.

7. Chubachi S, Yasuda H, Irie H, et al. A Case of NonSmall Cell Lung Cancer with Possible "Disease Flare" on Nivolumab Treatment. Case Rep Oncol Med 2016;2016:1075641.

8. Bernal Vaca L, Mendoza SD, Vergel JC, et al. Hyperprogression in Pediatric Melanoma Metastatic to the Breast Treated with a Checkpoint Inhibitor. Cureus 2019;11:e3859.

9. Chan AS, Ng VY, Snider J, et al. Hyperprogression of Liver Metastasis With Neoadjuvant Immunotherapy for Soft Tissue Sarcoma. Cureus 2020;12:e8575.

10. Fricke J, Mambetsariev I, Pharaon R, et al. Hyperprogression on immunotherapy with complete response to chemotherapy in a NSCLC patient with high PD-L1 and STK11: A case report. Medicine (Baltimore) 2020;99:e22323.

11. Wang W, Wu M, Liu M, et al. Hyperprogression to camrelizumab in a patient with esophageal squamous cell carcinoma harboring EGFR kinase domain duplication. J Immunother Cancer 2020;8:e000793.

12. Seymour L, Bogaerts J, Perrone A, et al. iRECIST: guidelines for response criteria for use in trials testing immunotherapeutics. Lancet Oncol 2017;18:e143-52.

13. Wolchok JD, Hoos A, O'Day S, et al. Guidelines for the evaluation of immune therapy activity in solid tumors: immune-related response criteria. Clin Cancer Res 2009;15:7412-20.

14. Zang H, Peng J, Zheng H, et al. Hyperprogression After Immune-Checkpoint Inhibitor Treatment: Characteristics and Hypotheses. Front Oncol 2020;10:515.
15. Eisenhauer EA, Therasse P, Bogaerts J, et al. New response evaluation criteria in solid tumours: revised RECIST guideline (version 1.1). Eur J Cancer 2009;45:228-47.

16. Nishino M, Giobbie-Hurder A, Gargano M, et al. Developing a common language for tumor response to immunotherapy: immune-related response criteria using unidimensional measurements. Clin Cancer Res 2013;19:3936-43.

17. Hodi FS, Ballinger M, Lyons B, et al. ImmuneModified Response Evaluation Criteria In Solid Tumors (imRECIST): Refining Guidelines to Assess the Clinical Benefit of Cancer Immunotherapy. J Clin Oncol 2018;36:850-8.

18. Kanjanapan Y, Day D, Wang L, et al. Hyperprogressive disease in early-phase immunotherapy trials: Clinical predictors and association with immune-related toxicities. Cancer 2019;125:1341-9.

19. Saâda-Bouzid E, Defaucheux C, Karabajakian A, et al. Hyperprogression during anti-PD-1/PD-L1 therapy in patients with recurrent and/or metastatic head and neck squamous cell carcinoma. Ann Oncol 2017;28:1605-11.

20. Ferrara R, Mezquita L, Texier M, et al. Hyperprogressive Disease in Patients With Advanced Non-Small Cell Lung Cancer Treated With PD-1/PD-L1 Inhibitors or With Single-Agent Chemotherapy. JAMA Oncol 2018;4:1543-52.

21. Kurman JS, Murgu SD. Hyperprogressive disease in patients with non-small cell lung cancer on immunotherapy. J Thorac Dis 2018;10:1124-8.

22. Kato S, Kurzrock R. Genomics of ImmunotherapyAssociated Hyperprogressors-Response. Clin Cancer Res 2017;23:6376

23. Lo Russo G, Moro M, Sommariva M, et al. Antibody$\mathrm{Fc} / \mathrm{FcR}$ Interaction on Macrophages as a Mechanism for Hyperprogressive Disease in Non-small Cell Lung Cancer Subsequent to PD-1/PD-L1 Blockade. Clin Cancer Res 2019;25:989-99.

24. Gandara DR, Reck M, Morris S, et al. Fast progression in patients treated with a checkpoint inhibitor (cpi) vs chemotherapy in OAK, a phase III trial of atezolizumab (atezo) vs docetaxel (doc) in 2L+ NSCLC. Ann Oncol 2018;29:x39-x43.

25. Liu C, Piao J, Shang Z. Hyperprogressive disease after radiotherapy combined with anti-PD-1 therapy in renal cell carcinoma: a case report and review of the literature. BMC Urol 2021;21:42.

26. Popat S. Hyperprogression with immunotherapy: Is it real? Cancer 2019;125:1218-20.

27. Kamada T, Togashi Y, Tay C, et al. PD-1+ regulatory 
T cells amplified by PD-1 blockade promote hyperprogression of cancer. Proc Natl Acad Sci U S A 2019;116:9999-10008.

28. Kim CG, Kim KH, Pyo KH, et al. Hyperprogressive disease during PD-1/PD-L1 blockade in patients with non-small-cell lung cancer. Ann Oncol 2019;30:1104-13.

29. Kocikowski M, Dziubek K, Parys M. Hyperprogression Under Immune Checkpoint-Based ImmunotherapyCurrent Understanding, The Role of PD-1/PDL1 Tumour-Intrinsic Signalling, Future Directions and a Potential Large Animal Model. Cancers (Basel) 2020;12:804.

30. Kato S, Goodman A, Walavalkar V, et al. Hyperprogressors after Immunotherapy: Analysis of Genomic Alterations Associated with Accelerated Growth Rate. Clin Cancer Res 2017;23:4242-50.

31. Refae S, Gal J, Brest P, et al. Hyperprogression under Immune Checkpoint Inhibitor: a potential role for germinal immunogenetics. Sci Rep 2020;10:3565.

32. Tawbi AHH, Burgess MA, Crowley J, et al. Safety and efficacy of PD-1 blockade using pembrolizumab in patients with advanced soft tissue (STS) and bone sarcomas (BS): Results of SARC028-A multicenter phase II study. J Clin Oncol 2016;34:abstr 11006.

33. Ye F, Tang C, Shi W, et al. A MDM2-dependent positive-feedback loop is involved in inhibition of miR375 and miR-106b induced by Helicobacter pylori lipopolysaccharide. Int J Cancer 2015;136:2120-31.

34. Kiriu T, Yamamoto M, Nagano T, et al. The time-series behavior of neutrophil-to-lymphocyte ratio is useful as a predictive marker in non-small cell lung cancer. PLoS One 2018;13:e0193018.

35. Sasaki A, Nakamura Y, Mishima S, et al. Predictive factors for hyperprogressive disease during nivolumab as antiPD1 treatment in patients with advanced gastric cancer. Gastric Cancer 2019;22:793-802.

36. Liu J, Wu Q, Wu S, et al. Investigation on potential biomarkers of hyperprogressive disease (HPD) triggered by immune checkpoint inhibitors (ICIs). Clin Transl Oncol 2021;23:1782-93.

37. Araujo DV, Wang A, Torti D, et al. Applications of Circulating Tumor DNA in a Cohort of Phase I Solid Tumor Patients Treated With Immunotherapy. JNCI Cancer Spectr 2021;5:pkaa122.

38. Wang Z, Duan J, Cai S, et al. Assessment of Blood Tumor Mutational Burden as a Potential Biomarker for Immunotherapy in Patients With Non-Small Cell Lung Cancer With Use of a Next-Generation Sequencing
Cancer Gene Panel. JAMA Oncol 2019;5:696-702.

39. Pessoa LS, Heringer M, Ferrer VP. ctDNA as a cancer biomarker: A broad overview. Crit Rev Oncol Hematol 2020;155:103109.

40. Lee JH, Long GV, Menzies AM, et al. Association Between Circulating Tumor DNA and Pseudoprogression in Patients With Metastatic Melanoma Treated With Anti-Programmed Cell Death 1 Antibodies. JAMA Oncol 2018;4:717-21.

41. Weiss GJ, Beck J, Braun DP, et al. Tumor Cell-Free DNA Copy Number Instability Predicts Therapeutic Response to Immunotherapy. Clin Cancer Res 2017;23:5074-81.

42. Ji Z, Cui Y, Peng Z, et al. Use of Radiomics to Predict Response to Immunotherapy of Malignant Tumors of the Digestive System. Med Sci Monit 2020;26:e924671.

43. Lambin P, Leijenaar RTH, Deist TM, et al. Radiomics: the bridge between medical imaging and personalized medicine. Nat Rev Clin Oncol 2017;14:749-62.

44. Sun R, Limkin EJ, Vakalopoulou M, et al. A radiomics approach to assess tumour-infiltrating CD8 cells and response to anti-PD-1 or anti-PD-L1 immunotherapy: an imaging biomarker, retrospective multicohort study. Lancet Oncol 2018;19:1180-91.

45. Gandara DR, von Pawel J, Mazieres J, et al. Atezolizumab Treatment Beyond Progression in Advanced NSCLC: Results From the Randomized, Phase III OAK Study. J Thorac Oncol 2018;13:1906-18.

46. Tunali I, Gray JE, Qi J, et al. Novel clinical and radiomic predictors of rapid disease progression phenotypes among lung cancer patients treated with immunotherapy: An early report. Lung Cancer 2019;129:75-9.

47. Ji Z, Peng Z, Gong J, et al. Hyperprogression after immunotherapy in patients with malignant tumors of digestive system. BMC Cancer 2019;19:705.

48. Matos I, Martin-Liberal J, García-Ruiz A, et al. Capturing Hyperprogressive Disease with Immune-Checkpoint Inhibitors Using RECIST 1.1 Criteria. Clin Cancer Res 2020;26:1846-55.

49. Ohue Y, Nishikawa H. Regulatory T (Treg) cells in cancer: Can Treg cells be a new therapeutic target? Cancer Sci 2019;110:2080-9.

50. Liu Y, Carlsson R, Comabella M, et al. FoxA1 directs the lineage and immunosuppressive properties of a novel regulatory $T$ cell population in EAE and MS. Nat Med 2014;20:272-82.

51. Chevalier MF, Schneider AK, Cesson V, et al. Conventional and PD-L1-expressing Regulatory T Cells are Enriched During BCG Therapy and may Limit its 
Efficacy. Eur Urol 2018;74:540-4.

52. Xiong D, Wang Y, Singavi AK, et al. Immunogenomic Landscape Contributes to Hyperprogressive Disease after Anti-PD-1 Immunotherapy for Cancer. iScience 2018;9:258-77.

53. Koukourakis MI, Giatromanolaki A, Sivridis E, et al. Lactate dehydrogenase-5 (LDH-5) overexpression in nonsmall-cell lung cancer tissues is linked to tumour hypoxia, angiogenic factor production and poor prognosis. $\mathrm{Br} \mathrm{J}$ Cancer 2003;89:877-85.

54. Schindler C, Levy DE, Decker T. JAK-STAT signaling: from interferons to cytokines. J Biol Chem 2007;282:20059-63.

55. Zhou S, Gu L, He J, et al. MDM2 regulates vascular endothelial growth factor mRNA stabilization in hypoxia. Mol Cell Biol 2011;31:4928-37.

56. Akbay EA, Koyama S, Carretero J, et al. Activation of the PD-1 pathway contributes to immune escape in EGFR-

Cite this article as: Lin M, Vanneste BGL, Yu Q, Chen Z, Peng J, Cai X. Hyperprogression under immunotherapy: a new form of immunotherapy response? - a narrative literature review. Transl Lung Cancer Res 2021;10(7):3276-3291. doi: 10.21037/ tlcr-21-575 driven lung tumors. Cancer Discov 2013;3:1355-63.

57. Du S, McCall N, Park K, et al. Blockade of TumorExpressed PD-1 promotes lung cancer growth. Oncoimmunology 2018;7:e1408747.

58. Knorr DA, Dahan R, Ravetch JV. Toxicity of an Fcengineered anti-CD40 antibody is abrogated by intratumoral injection and results in durable antitumor immunity. Proc Natl Acad Sci U S A 2018;115:11048-53.

59. Dahan R, Sega E, Engelhardt J, et al. FcyRs Modulate the Anti-tumor Activity of Antibodies Targeting the PD-1/ PD-L1 Axis. Cancer Cell 2015;28:285-95.

60. Zhang T, Song X, Xu L, et al. The binding of an antiPD-1 antibody to FcyRI has a profound impact on its biological functions. Cancer Immunol Immunother 2018;67:1079-90.

61. Knorr DA, Ravetch JV. Immunotherapy and Hyperprogression: Unwanted Outcomes, Unclear Mechanism. Clin Cancer Res 2019;25:904-6. 